\title{
Sedative load of medications prescribed for older people with dementia in care homes
}

\author{
Carole Parsons ${ }^{1 *}$, Jane Haydock , Elspeth Mathie ${ }^{2}$, Natasha Baron³ ${ }^{3}$ Ina Machen², Elizabeth Stevenson², \\ Sarah Amador ${ }^{2}$ and Claire Goodman ${ }^{2}$
}

\begin{abstract}
Background: The objective of this study was to determine the sedative load and use of sedative and psychotropic medications among older people with dementia living in (residential) care homes.

Methods: Medication data were collected at baseline and at two further time-points for eligible residents of six care homes participating in the EVIDEM-End Of Life (EOL) study for whom medication administration records were available. Regular medications were classified using the Anatomical Therapeutic Chemical classification system and individual sedative loads were calculated using a previously published model.
\end{abstract}

Results: At baseline, medication administration records were reviewed for 115 residents; medication records were reviewed for 112 and 105 residents at time-points 2 and 3 respectively. Approximately one-third of residents were not taking any medications with sedative properties at each time-point, while a significant proportion of residents had a low sedative load score of 1 or $2(54.8 \%, 59.0 \%$ and $57.1 \%$ at baseline and time-points 2 and 3 respectively). More than $10 \%$ of residents had a high sedative load score $(\geq 3)$ at baseline $(12.2 \%)$, and this increased to $14.3 \%$ at time-points 2 and 3. Approximately two-thirds of residents (66.9\%) regularly used one or more psychotropic medication(s). Antidepressants, predominantly selective serotonin re-uptake inhibitors (SSRIs), were most frequently used, while antipsychotics, hypnotics and anxiolytics were less routinely administered. The prevalence of antipsychotic use among residents was 19.0\%, lower than has been previously reported for nursing home residents. Throughout the duration of the study, administration of medications recognised as having prominent sedative adverse effects and/or containing sedative components outweighed the regular use of primary sedatives.

Conclusions: Sedative load scores were similar throughout the study period for residents with dementia in each of the care homes. Scores were lower than previously reported in studies conducted in long-term care wards which have on-site clinical support. Nevertheless, strategies to optimise drug therapy for care home residents with dementia which rely on clinicians external to the care home for support and medication review are required.

\section{Background}

In the United Kingdom (UK), care homes are the main providers of long-term care for older people. They include care homes which provide 24-hour nursing care (nursing homes), those that provide personal care only (residential homes), and those with mixed provision, which offer both residential and nursing care [1]. Residential homes, which provide the majority of long-term care for older people in England, do not have on-site nursing provision, and rely on primary healthcare professionals, namely general

\footnotetext{
*Correspondence: c.parsons@qub.ac.uk

'School of Pharmacy, Queen's University Belfast, 97 Lisburn Road, Belfast, BT9 7BL, Northern Ireland, UK

Full list of author information is available at the end of the article
}

practitioners (GPs), nurses and community pharmacists, for meeting healthcare needs, including prescribing and supply of medications and medication review [2].

Prescribing is one of the most common medical interventions experienced by older people resident in care homes $[3,4]$. Due to multiple medical conditions and polypharmacy (defined as the use of multiple medications and/or administration of more medications than are clinically indicated [5]), in addition to age-related changes in pharmacokinetics and pharmacodynamics, care home residents are at high risk of adverse drug events (ADEs) [6-8]. Older people with cognitive impairment are particularly susceptible to ADEs associated with sedative and psychotropic drugs [9]. The over-use of

\section{() Biomed Central}


psychotropic drugs (antipsychotics, antidepressants, hypnotics, and anxiolytics) has been a particular concern in the literature on prescribing and use of medicines in care homes [10-17]; however, nursing homes have been the primary focus and these studies have not been limited to residents with cognitive impairment. Furthermore previous studies which compare psychotropic drug use among patients with and without dementia have been limited to specific classes of psychotropic drugs [18-21]. Recent interest has focused on the development of a measure to quantify residents' overall drug load [22-32]. Sedative drugs may include medications prescribed for intentional sedation, medications with which sedation is a prominent side-effect, or medications which yield sedation as a potential ADE [33,34]. A model has been developed to quantify the cumulative effect of taking multiple drugs with sedative properties, termed the sedative load $[35,36]$. This model has been utilised thus far to examine the sedative load among residents of long-term care wards in Finland $[33,34]$. An alternative model, the Drug Burden Index (a measure of a person's exposure to anticholinergic and sedative medications), has been used to examine exposure of residents of residential aged-care facilities in Australia to medications with anticholinergic and sedative properties $[27,37,38]$. However, to the best of the authors' knowledge, no studies have been conducted to date which use the sedative load model to examine prescribing of sedative medications in residents of residential homes. The objective of this study was to determine the sedative load and use of sedative and psychotropic medications among older people with dementia living in residential care homes.

\section{Methods}

The eligible study population comprised all residents from six residential care homes participating in the EVIDEM-End Of Life (EOL) study for whom medication administration records were available at baseline and at the two further time-points (approximately sixteen weeks apart) at which data were collected over the 12-month data collection period. The EVIDEM-EOL study had a prospective design, tracking the events and care experienced by older people with dementia over two years, and was undertaken between April 2008 and June 2010. Ethical approval for the EVIDEM-EOL study was granted by the NHS Southampton and South West Hampshire Research Ethics Committee in July 2008 (MREC Ref: 08/ H0502/74). All six care homes were registered to provide dementia care. Care home residents who were 65 years of age or older, and who had a documented diagnosis of dementia, or who were determined by senior care home staff as having cognitive impairment consistent with a diagnosis of dementia, were eligible to participate in the study. Written informed consent to participate was obtained from those eligible residents who were considered to have capacity to consent. For those residents deemed not to have capacity to consent for themselves, written assent was obtained from a personal consultee who, based on his/her knowledge of the resident, could provide an opinion as to whether the resident would have consented to his/her care notes being reviewed. Residents with dementia whom the care home manager thought it inappropriate to approach (for example, residents in the terminal stage of the disease), or who lacked capacity to consent and for whom a consultee could not be identified, were excluded from the study [39].

Medication data for each participating resident were collected at baseline and at two further time-points using medication administration records obtained from the care home notes which detailed the medications prescribed and administered by care home staff over the four-week period which coincided with each data collection timepoint. The maximum number of medications prescribed for and administered to the resident at any one time during this four-week period was utilised to determine drug use at each data collection time-point. All medications were categorised using the Anatomical Therapeutic Chemical (ATC) classification system as recommended by the World Health Organisation [40]. As per previous research examining sedative load of medications [33,34], medications taken regularly were considered, while medications used on a pro re nata (prn) basis were excluded. Medications were considered to be regularly taken if there was a documented regular sequence of administration. Psychotropic medications were defined as antipsychotics (ATC classification N05A), antidepressants (N06A), hypnotics (N05C) and anxiolytics (N05B). The sedative load for each resident was calculated using the sedative load model which classifies medications into four groups (Group 1: primary sedatives; Group 2: medications with sedation as a prominent side-effect or preparations with a sedating component; Group 3: medications with sedation as a potential ADE; Group 4: all other medications with no known sedative properties). Medications in groups 1 and 2 were given sedative ratings of 2 and 1 respectively, and the overall sedative load for each resident was calculated using the following formula:

$$
\text { Sedative Loading }(\mathrm{SL})=\sum_{\mathrm{k}=1}^{\mathrm{n}} \mathrm{SR}_{\mathrm{k}}
$$

where: $\mathrm{n}=$ number of drugs and $\mathrm{SR}_{\mathrm{k}}=$ sedative rating for drug $\mathrm{k}$ [29].

Data were analysed using PASW Statistics 18.0 software (SPSS Inc. Chicago, Illinois). Chi-squared $(\chi 2)$ tests were used to compare categorical variables and KruskalWallis tests were used to compare continuous variables. Significance was set $a$ priori at $\mathrm{p} \leq 0.05$. 


\section{Results}

A total of 214 residents across the six care homes were eligible for participation in the EVIDEM-EOL study. Of these, 133 residents were recruited (62.2\%). Details of the recruitment of residential care homes and residents have been previously published [39].

At baseline, medication administration records for 115 residents (86.5\%) were reviewed; medication administration record sheets were unavailable for 18 residents as they were archived or otherwise unobtainable as the resident had died. The mean age of the residents was 85.8 (SD 6.8) years and 91 of these residents (79.1\%) were female; the demographic profile of residents included in the study is detailed in Table 1. Between two and four general practice surgeries provided services to each care home; care homes 1, 2, 5 and 6 were each served by two general practice surgeries, while care homes 3 and 4 were each served by four general practice surgeries.

At baseline, eighty-six residents (74.8\%) had a recorded diagnosis of dementia in their care home notes, although $33(38.4 \%)$ of these residents did not have a specific type of dementia recorded. Twenty-nine residents (25.2\%) did not have a diagnosis of dementia in their care home notes, but were determined by care home staff as having cognitive impairment consistent with a diagnosis of dementia. None of the care homes recorded severity of dementia in their care notes but behavioural and communication difficulties arising from having dementia were recorded. According to the care home notes, a specific cognitive test, such as the Mini Mental State Examination (MMSE [41]), was administered to 23 residents (20.0\%). Fifty residents (43.5\%) were recorded as having 3 or more co-morbidities (obtained from the care home notes), and the mean number of long-term conditions per resident was 2.46 (SD 1.46). The Cornell Scale for Depression in Dementia [42] was applied by the care home manager, or senior care home staff, for the 91 residents for whom the required data was available. Ten residents $(11.0 \%)$ scored 12 or more (indicating probable depression) and two of the care homes (3 and 4) did not identify symptoms of depression in any of their residents. Twenty-five residents (21.7\%) were recorded as having a diagnosis of depression in their care home notes.

The number of residents with sedative load scores of $0-6$ at each time-point are detailed in Table 2 . Approximately one-third of residents had a sedative load score of 0 (33.0\%, 26.8\% and 28.5\% at baseline and time-points 2 and 3 respectively), and a significant proportion of residents had a low sedative load score of 1 or $2(54.8 \%, 59.0 \%$ and $57.1 \%$ at baseline and timepoints 2 and 3 respectively), while $12.2 \%$ had a high sedative load $(\geq 3)$ at baseline, increasing slightly to $14.3 \%$ at time-points 2 and 3 .
Use of regular medications is outlined in Table 3. At baseline, the mean number of regular medications prescribed for and administered to each resident ranged from 4.36 (SD 2.11) in care home 4 to 7.41 (SD 4.83) in care home 2. Prevalence of polypharmacy, defined by the National Service Framework for Older People [43] as the prescription of four or more medications, varied within the study population; residents in care home 6 had significantly lower levels of polypharmacy than residents in care home 5. Approximately two-thirds of residents $(\mathrm{n}=77,66.9 \%)$ regularly used one or more psychotropic medication(s) [an antipsychotic, hypnotic, anxiolytic or antidepressant].

Data relating to individual drug classes showed variability across the study population, as presented in Table 4. The use of antipsychotic medications ranged from 10.0\% to $41.2 \%$ across care homes, with care home 2 found to have a considerably higher level of antipsychotic prescribing than the other homes. The most commonly used atypical antipsychotic was quetiapine $(\mathrm{n}=14,12.2 \%)$ as shown in Table 5. All residents taking antipsychotics in care home 1 were prescribed conventional antipsychotics (Tables 4 and 5).

Regular use of antidepressant medication ranged from $33.3 \%$ in care home 5 to $68.2 \%$ in care home 4 . Selective serotonin re-uptake inhibitors (SSRIs) were the most widely used class of antidepressants across all care homes with the exception of care home 4, where trazodone prescribing predominated. This finding is presented in Table 4, in which trazodone is categorised as 'other antidepressants'. Twenty-one of the 22 residents in care home 4 were assessed at baseline using the Cornell Scale for Depression in Dementia [42], and all of these residents were classified as unlikely to be suffering from depression (a Cornell score of less than 12), indicating that trazodone may have been prescribed for treatment of behavioural symptoms such as agitation. However, when assessed using criteria for agitated/not agitated status adapted from the Cohen-Mansfield Agitation Inventory [44], these residents did not show significantly higher scores for agitation than residents in the other care homes $(\chi 2$ test; $\mathrm{p}>0.05)$. This indicated that trazodone may have instead been used for treatment of insomnia. Trazodone was only prescribed for one other resident in care home 5, and was not prescribed for any of the ten residents in the study who were determined to be suffering from probable depression (a Cornell score equal to or more than 12). Citalopram was the most frequently prescribed SSRI in the care homes ( $\mathrm{n}=18,15.7 \%$; table 5).

Regular administration of hypnotics and anxiolytics was low, ranging from $5.0 \%$ in care home 1 to $21.4 \%$ in care home 3 , while the most frequently used hypnotic and anxiolytic medications were temazepam (3.5\%) and lorazepam (2.6\%) respectively (Table 5). Hypnotics were 
Table 1 Demographic profile for residential care home residents with dementia

\begin{tabular}{|c|c|c|c|c|c|c|c|c|c|c|c|c|c|c|c|c|c|c|}
\hline \multirow[t]{3}{*}{ Parameter } & \multicolumn{17}{|c|}{ Care home at each time point } & \multirow[b]{3}{*}{ TP3 } \\
\hline & \multicolumn{2}{|l|}{1} & \multicolumn{3}{|c|}{2} & \multicolumn{3}{|c|}{3} & \multicolumn{3}{|c|}{4} & \multicolumn{3}{|c|}{5} & \multicolumn{3}{|c|}{6} & \\
\hline & $\mathrm{BL}$ & TP2 & TP3 & BL & TP2 & TP3 & $\mathrm{BL}$ & TP2 & TP3 & BL & TP2 & TP3 & BL & TP2 & TP3 & $\mathrm{BL}$ & TP2 & \\
\hline $\begin{array}{l}\text { Number of } \\
\text { residents }(n)\end{array}$ & 20 & 20 & 19 & 17 & 17 & 16 & 14 & 14 & 14 & 22 & 22 & 21 & 30 & 27 & 25 & 12 & 12 & 10 \\
\hline \multicolumn{19}{|l|}{ Gender } \\
\hline Female [no.(\%)] & $18(90.0)$ & $18(90.0)$ & $17(89.5)$ & $14(82.4)$ & $14(82.4)$ & $13(81.3)$ & $12(85.7)$ & $12(85.7)$ & $12(85.7)$ & $20(90.9)$ & $20(90.9)$ & $20(95.2)$ & $19(63.3)$ & $17(63.0)$ & $18(72.0)$ & $8(66.7)$ & $8(66.7)$ & $7(70.0)$ \\
\hline Male [no.(\%)] & $2(10.0)$ & $2(10.0)$ & $2(10.5)$ & $3(17.6)$ & $3(17.7)$ & $3(18.7)$ & $2(14.3)$ & $2(14.3)$ & $2(14.3)$ & $2(9.1)$ & $2(9.1)$ & $1(4.8)$ & $11(36.7)$ & $10(37.0)$ & 7 (28.0) & $4(33.3)$ & $4(33.3)$ & $3(30.0)$ \\
\hline \multicolumn{19}{|l|}{ Age } \\
\hline Mean & 87.4 & 87.7 & 88.4 & 87.3 & 88.6 & 87.9 & 89.0 & 89.9 & 90.4 & 84.6 & 85.7 & 85.9 & 83.5 & 83.9 & 83.2 & 83.7 & 84.0 & 84.9 \\
\hline $\begin{array}{l}\text { Standard } \\
\text { deviation }\end{array}$ & 6.16 & 6.14 & 6.25 & 7.56 & 8.81 & 7.75 & 5.87 & 5.70 & 5.62 & 6.63 & 5.87 & 5.97 & 6.63 & 6.88 & 6.21 & 8.28 & 8.28 & 8.53 \\
\hline
\end{tabular}

BL - baseline, TP - time point. 
Table 2 Sedative load for residential care home residents with dementia

\begin{tabular}{|c|c|c|c|c|c|c|c|c|c|c|c|c|c|c|c|c|c|c|c|c|c|}
\hline \multirow[b]{3}{*}{ Sedative Load } & \multicolumn{18}{|c|}{ Number of residents with sedative load at each time point } & & & \\
\hline & \multicolumn{3}{|c|}{ Care Home 1} & \multicolumn{3}{|c|}{ Care Home 2} & \multicolumn{3}{|c|}{ Care Home 3} & \multicolumn{3}{|c|}{ Care Home 4} & \multicolumn{3}{|c|}{ Care Home 5} & \multicolumn{3}{|c|}{ Care Home 6} & \multicolumn{3}{|c|}{ Total (\%) } \\
\hline & $\mathrm{BL}$ & TP 2 & TP 3 & BL & TP 2 & TP 3 & $\mathrm{BL}$ & TP 2 & TP 3 & $\mathrm{BL}$ & TP 2 & TP 3 & $\mathrm{BL}$ & TP 2 & TP 3 & $B L$ & TP 2 & TP 3 & $\mathrm{BL}$ & TP 2 & TP 3 \\
\hline 0 & 8 & 6 & 4 & 3 & 4 & 7 & 5 & 5 & 6 & 6 & 4 & 5 & 12 & 10 & 7 & 4 & 1 & 1 & $38(33.0)$ & $30(26.8)$ & $30(28.5)$ \\
\hline 1 & 8 & 7 & 8 & 11 & 9 & 7 & 5 & 4 & 4 & 5 & 6 & 5 & 8 & 9 & 9 & 4 & 7 & 5 & $41(35.7)$ & $42(37.5)$ & $38(36.2)$ \\
\hline 2 & 1 & 3 & 3 & 1 & 3 & 1 & 1 & 2 & 0 & 9 & 9 & 9 & 8 & 5 & 5 & 2 & 2 & 4 & $22(19.1)$ & $24(21.4)$ & $22(21.0)$ \\
\hline 3 & 2 & 3 & 3 & 1 & 1 & 1 & 2 & 1 & 2 & 2 & 3 & 2 & 2 & 2 & 2 & 2 & 2 & 0 & $11(9.6)$ & $12(10.7)$ & $10(9.5)$ \\
\hline 4 & 0 & 0 & 0 & 0 & 0 & 0 & 1 & 2 & 1 & 0 & 0 & 0 & 0 & 1 & 2 & 0 & 0 & 0 & $1(0.9)$ & $3(2.7)$ & $3(2.9)$ \\
\hline 5 & 1 & 1 & 1 & 0 & 0 & 0 & 0 & 0 & 1 & 0 & 0 & 0 & 0 & 0 & 0 & 0 & 0 & 0 & $1(0.9)$ & $1(0.9)$ & $2(1.9)$ \\
\hline 6 & 0 & 0 & 0 & 1 & 0 & 0 & 0 & 0 & 0 & 0 & 0 & 0 & 0 & 0 & 0 & 0 & 0 & 0 & $1(0.9)$ & $0(0)$ & $0(0)$ \\
\hline Total & 20 & 20 & 19 & 17 & 17 & 16 & 14 & 14 & 14 & 22 & 22 & 21 & 30 & 27 & 25 & 12 & 12 & 10 & $115(100.0)$ & $112(100.0)$ & $105(100.0)$ \\
\hline
\end{tabular}

BL - baseline, TP - time point 
Table 3 Use of regular medications, including sedative medications, by residential care home residents with dementia

\begin{tabular}{|c|c|c|c|c|c|c|c|c|c|c|c|c|c|c|c|c|c|c|c|c|c|}
\hline \multirow[t]{3}{*}{ Parameter } & \multicolumn{17}{|c|}{ Care home at each time point } & \multicolumn{4}{|c|}{$p$ - Value } \\
\hline & \multicolumn{2}{|l|}{1} & \multicolumn{3}{|c|}{2} & \multicolumn{3}{|c|}{3} & \multicolumn{3}{|c|}{4} & \multicolumn{3}{|c|}{5} & \multicolumn{3}{|c|}{6} & \multirow[b]{2}{*}{ TP3 } & \multirow[b]{2}{*}{ BL } & \multirow[b]{2}{*}{ TP2 } & \multirow[b]{2}{*}{ TP3 } \\
\hline & BL & TP2 & TP3 & BL & TP2 & TP3 & BL & TP2 & TP3 & BL & TP2 & TP3 & BL & TP2 & TP3 & BL & TP2 & & & & \\
\hline Number of residents $(n)$ & 20 & 20 & 19 & 17 & 17 & 16 & 14 & 14 & 14 & 22 & 22 & 21 & 30 & 27 & 25 & 12 & 12 & 10 & & & \\
\hline \multicolumn{22}{|l|}{$\begin{array}{l}\text { Number of regular } \\
\text { medications }\end{array}$} \\
\hline Mean & 6.70 & 5.10 & 5.11 & 7.41 & 5.88 & 6.00 & 5.79 & 5.57 & 5.57 & 4.36 & 4.41 & 4.33 & 6.17 & 6.74 & 6.88 & 5.17 & 5.75 & 5.90 & 0.07 & 0.13 & 0.10 \\
\hline Range & $1-10$ & $1-8$ & $1-8$ & $2-18$ & $2-11$ & $2-11$ & $1-10$ & $0-13$ & $0-13$ & $0-8$ & $0-9$ & $1-9$ & $0-10$ & $0-13$ & $0-13$ & $0-12$ & $0-12$ & $1-12$ & & & \\
\hline Standard deviation & 3.18 & 2.40 & 2.47 & 4.83 & 3.06 & 3.12 & 3.17 & 3.28 & 3.28 & 2.11 & 2.32 & 2.35 & 2.51 & 3.58 & 3.59 & 3.86 & 3.14 & 3.51 & & & \\
\hline $\begin{array}{l}\text { Polypharmacy (>4 } \\
\text { medications) [no.(\%)] }\end{array}$ & $\begin{array}{c}16 \\
(80.0)\end{array}$ & $\begin{array}{c}18 \\
(90.0)\end{array}$ & $\begin{array}{c}14 \\
(73.6)\end{array}$ & $\begin{array}{c}15 \\
(88.2)\end{array}$ & $\begin{array}{c}14 \\
(82.3)\end{array}$ & $\begin{array}{c}12 \\
(75.0)\end{array}$ & $\begin{array}{c}9 \\
(64.2)\end{array}$ & $\begin{array}{c}5 \\
(35.7)\end{array}$ & $\begin{array}{c}10 \\
(74.1)\end{array}$ & $\begin{array}{c}14 \\
(63.6)\end{array}$ & $\begin{array}{c}16 \\
(72.7)\end{array}$ & $\begin{array}{c}11 \\
(52.4)\end{array}$ & $\begin{array}{c}25 \\
(83.3)\end{array}$ & $\begin{array}{c}20 \\
(74.0)\end{array}$ & $\begin{array}{c}23 \\
(92.0)\end{array}$ & $\begin{array}{c}7 \\
(58.3)\end{array}$ & $\begin{array}{c}5 \\
(41.7)\end{array}$ & $\begin{array}{c}9 \\
(90.0)\end{array}$ & 0.02 & 0.19 & 0.08 \\
\hline \multicolumn{22}{|l|}{ Sedative Load } \\
\hline Mean & 1.05 & 1.35 & 1.47 & 1.29 & 1.06 & 0.75 & 1.21 & 1.36 & 1.36 & 1.32 & 1.50 & 1.38 & 1.03 & 1.07 & 1.32 & 1.08 & 1.42 & 1.30 & 0.84 & 0.59 & 0.39 \\
\hline Range & $0-5$ & $0-5$ & $0-5$ & $0-6$ & $0-3$ & $0-3$ & $0-4$ & $0-4$ & $0-5$ & $0-3$ & $0-3$ & $0-3$ & $0-3$ & $0-4$ & $0-4$ & $0-3$ & $0-3$ & $0-2$ & & & \\
\hline Standard deviation & 1.32 & 1.35 & 1.31 & 1.40 & 0.83 & 0.86 & 1.31 & 1.45 & 1.69 & 0.99 & 0.96 & 0.97 & 0.98 & 1.11 & 1.22 & 1.12 & 0.9 & 0.68 & & & \\
\hline Antipsychotics [no.(\%)] & $\begin{array}{c}2 \\
(10.0)\end{array}$ & $\begin{array}{c}4 \\
(20.0)\end{array}$ & $\begin{array}{c}5 \\
(26.3)\end{array}$ & $\begin{array}{c}7 \\
(41.2)\end{array}$ & $\begin{array}{c}6 \\
(35.3)\end{array}$ & $\begin{array}{c}4 \\
(25.0)\end{array}$ & $\begin{array}{c}2 \\
(14.3)\end{array}$ & $\begin{array}{c}1 \\
(7.1)\end{array}$ & $1(7.1)$ & $\begin{array}{c}7 \\
(32.0)\end{array}$ & $\begin{array}{c}6 \\
(27.3)\end{array}$ & $\begin{array}{c}5 \\
(23.8)\end{array}$ & $\begin{array}{c}3 \\
(10.0)\end{array}$ & $\begin{array}{c}3 \\
(11.1)\end{array}$ & $\begin{array}{c}4 \\
(16.0)\end{array}$ & $\begin{array}{c}2 \\
(16.8)\end{array}$ & $\begin{array}{c}1 \\
(8.3)\end{array}$ & 0 & 0.02 & 0.17 & 0.04 \\
\hline Antidepressants [no.(\%)] & $\begin{array}{c}11 \\
(55.0)\end{array}$ & $\begin{array}{c}12 \\
(60.0)\end{array}$ & $\begin{array}{c}11 \\
(57.9)\end{array}$ & $\begin{array}{c}9 \\
(52.9)\end{array}$ & $\begin{array}{c}6 \\
(35.3)\end{array}$ & $\begin{array}{c}4 \\
(25.0)\end{array}$ & $\begin{array}{c}5 \\
(35.7)\end{array}$ & $\begin{array}{c}8 \\
(57.1)\end{array}$ & $\begin{array}{c}9 \\
(64.3)\end{array}$ & $\begin{array}{c}15 \\
(68.2)\end{array}$ & $\begin{array}{c}14 \\
(63.6)\end{array}$ & $\begin{array}{c}13 \\
(61.9)\end{array}$ & $\begin{array}{c}10 \\
(33.3)\end{array}$ & $\begin{array}{c}11 \\
(40.7)\end{array}$ & $\begin{array}{c}11 \\
(44.0)\end{array}$ & $\begin{array}{c}4 \\
(33.3)\end{array}$ & $\begin{array}{c}6 \\
(50.0)\end{array}$ & $\begin{array}{c}6 \\
(60.0)\end{array}$ & 0.29 & 0.41 & 0.35 \\
\hline $\begin{array}{l}\text { Hypnotics and anxiolytics [no. } \\
(\%)]\end{array}$ & $1(5.0)$ & $1(5.0)$ & $1(5.3)$ & $1(5.9)$ & 0 & 0 & $\begin{array}{c}3 \\
(21.4)\end{array}$ & $\begin{array}{c}3 \\
(21.4)\end{array}$ & $\begin{array}{c}2 \\
(14.3)\end{array}$ & $2(9.1)$ & $2(9.1)$ & $2(9.5)$ & $\begin{array}{c}4 \\
(13.3)\end{array}$ & $\begin{array}{c}5 \\
(18.5)\end{array}$ & $\begin{array}{c}5 \\
(20.0)\end{array}$ & $\begin{array}{c}2 \\
(16.7)\end{array}$ & $\begin{array}{c}3 \\
(25.0)\end{array}$ & $\begin{array}{c}2 \\
(20.0)\end{array}$ & 0.06 & 0.14 & 0.23 \\
\hline
\end{tabular}

BL - baseline, TP - time point 
Table 4 Use of classes of psychotropic medications by residential care home residents with dementia

\section{Drug Class \\ Care home data at each time point}

\begin{tabular}{|c|c|c|c|c|c|c|c|c|c|c|c|c|c|c|c|c|c|c|}
\hline & \multicolumn{3}{|c|}{1} & \multicolumn{3}{|c|}{2} & \multicolumn{3}{|c|}{3} & \multicolumn{3}{|c|}{4} & \multicolumn{3}{|c|}{5} & \multicolumn{3}{|c|}{6} \\
\hline & $B L$ & TP 2 & TP 3 & $B L$ & TP 2 & TP 3 & $B L$ & TP 2 & TP 3 & $\mathrm{BL}$ & TP 2 & TP 3 & BL & TP 2 & TP 3 & $B L$ & TP 2 & TP 3 \\
\hline \multicolumn{19}{|l|}{ Antipsychotics } \\
\hline Conventional [no.(\%)] & $2(10.0)$ & $4(20.0)$ & $5(26.3)$ & 0 & 0 & 0 & $1(7.1)$ & $1(7.1)$ & $1(7.1)$ & $1(4.6)$ & $1(4.6)$ & $1(4.8)$ & $1(3.3)$ & $1(3.7)$ & $2(8.0)$ & $1(8.3)$ & $1(8.3)$ & 0 \\
\hline Atypical [no.(\%)] & 0 & 0 & 0 & $\begin{array}{c}7 \\
(41.2)\end{array}$ & $\begin{array}{c}6 \\
(35.3)\end{array}$ & $\begin{array}{c}4 \\
(25.0)\end{array}$ & $1(7.1)$ & 0 & 0 & $6(27.3)$ & $5(22.7)$ & $4(19.0)$ & $2(6.7)$ & $2(7.4)$ & $2(8.0)$ & $1(8.3)$ & 0 & 0 \\
\hline \multicolumn{19}{|l|}{ Antidepressants } \\
\hline TCAs [no.(\%)] & 0 & $1(5.0)$ & 0 & $\begin{array}{c}3 \\
(17.7)\end{array}$ & $1(5.9)$ & $1(6.3)$ & $1(7.1)$ & $1(7.1)$ & $1(7.1)$ & $1(4.6)$ & 0 & 0 & 0 & 0 & $1(4.0)$ & 0 & 0 & 0 \\
\hline SSRIs [no.(\%)] & $\begin{array}{c}11 \\
(55.0)\end{array}$ & $\begin{array}{c}11 \\
(55.0)\end{array}$ & $\begin{array}{c}10 \\
(52.6)\end{array}$ & $\begin{array}{c}6 \\
(35.3)\end{array}$ & $\begin{array}{c}5 \\
(29.4)\end{array}$ & $\begin{array}{c}3 \\
(18.8)\end{array}$ & $\begin{array}{c}4 \\
(28.6)\end{array}$ & $\begin{array}{c}4 \\
(28.6)\end{array}$ & $\begin{array}{c}5 \\
(35.7)\end{array}$ & $2(9.1)$ & $2(9.1)$ & $2(9.5)$ & $\begin{array}{c}7 \\
(23.3)\end{array}$ & $\begin{array}{c}9 \\
(33.3)\end{array}$ & $\begin{array}{c}7 \\
(28.0)\end{array}$ & $\begin{array}{c}3 \\
(25.0)\end{array}$ & $\begin{array}{c}5 \\
(41.7)\end{array}$ & $\begin{array}{c}5 \\
(50.0)\end{array}$ \\
\hline $\begin{array}{l}\text { Other antidepressants [no. } \\
(\%)]\end{array}$ & 0 & 0 & $1(5.3)$ & 0 & 0 & $1(6.3)$ & 0 & $\begin{array}{c}3 \\
(21.4)\end{array}$ & $\begin{array}{c}3 \\
(21.4)\end{array}$ & $\begin{array}{c}12 \\
(54.6)\end{array}$ & $\begin{array}{c}12 \\
(54.6)\end{array}$ & $\begin{array}{c}11 \\
(52.4)\end{array}$ & $\begin{array}{c}3 \\
(10.0)\end{array}$ & $2(7.4)$ & $\begin{array}{c}3 \\
(12.0)\end{array}$ & $1(8.3)$ & $1(8.3)$ & $\begin{array}{c}1 \\
(10.0)\end{array}$ \\
\hline Hypnotics [no.(\%)] & $1(5.0)$ & $1(5.0)$ & $1(5.3)$ & 0 & 0 & 0 & $\begin{array}{c}2 \\
(14.3)\end{array}$ & $\begin{array}{c}2 \\
(14.3)\end{array}$ & $1(7.1)$ & $1(4.6)$ & $1(4.6)$ & $1(4.8)$ & $2(6.7)$ & $2(7.4)$ & $2(8.0)$ & $\begin{array}{c}2 \\
(16.7)\end{array}$ & $\begin{array}{c}3 \\
(25.0)\end{array}$ & $\begin{array}{c}2 \\
(20.0)\end{array}$ \\
\hline Anxiolytics [no.(\%)] & 0 & 0 & 0 & $1(5.9)$ & 0 & 0 & $1(7.1)$ & $1(7.1)$ & $1(7.1)$ & $1(4.6)$ & $1(4.6)$ & $1(4.8)$ & $2(6.7)$ & $\begin{array}{c}3 \\
(11.1)\end{array}$ & $\begin{array}{c}3 \\
(12.0)\end{array}$ & 0 & 0 & 0 \\
\hline
\end{tabular}

BL - baseline, TP - time point

TCA - tricyclic antidepressant; SSRI - selective serotonin re-uptake inhibitor 
Table 5 Prevalence of the four most commonly used primary sedatives and drugs with sedation as a prominent adverse effect/containing a sedating component among residential care home residents with dementia

\begin{tabular}{|c|c|c|c|c|c|c|c|c|}
\hline \multirow[t]{3}{*}{ Variable } & \multirow[t]{3}{*}{ Drug Name } & \multirow{3}{*}{$\begin{array}{l}\text { All residents } \\
\qquad n=115\end{array}$} & \multicolumn{6}{|c|}{ Care Home } \\
\hline & & & 1 & 2 & 3 & 4 & 5 & 6 \\
\hline & & & $\mathrm{n}=\mathbf{2 0}$ & $\mathrm{n}=17$ & $n=14$ & $\mathrm{n}=\mathbf{2 2}$ & $\mathrm{n}=30$ & $n=12$ \\
\hline \multicolumn{9}{|l|}{ Baseline } \\
\hline \multirow[t]{4}{*}{ Primary Sedatives [no.(\%)] } & Promazine & $5(4.4)$ & $2(10.0)$ & 0 & $1(7.1)$ & $1(4.5)$ & $1(3.3)$ & 0 \\
\hline & Temazepam & $4(3.5)$ & 0 & 0 & $1(7.1)$ & $1(4.5)$ & 0 & $1(8.3)$ \\
\hline & Lorazepam & $3(2.6)$ & 0 & $1(5.9)$ & $1(7.1)$ & $1(4.5)$ & 0 & 0 \\
\hline & Amitriptyline & $3(2.6)$ & $2(10.0)$ & $1(5.9)$ & 0 & 0 & 0 & 0 \\
\hline \multirow{4}{*}{$\begin{array}{l}\text { Drugs with sedation as a prominent adverse effect/ } \\
\text { containing a sedating component [no.(\%)] }\end{array}$} & Citalopram & $18(15.7)$ & $6(30.0)$ & $2(11.8)$ & $1(7.1)$ & $1(4.5)$ & $5(16.7)$ & $3(25.0)$ \\
\hline & Quetiapine & $14(12.2)$ & 0 & $6(35.3)$ & $1(7.1)$ & $5(22.7)$ & $1(3.3)$ & $1(8.3)$ \\
\hline & Trazodone & $12(10.4)$ & 0 & 0 & 0 & $11(50.0)$ & $1(3.3)$ & 0 \\
\hline & Fluoxetine & $8(7.0)$ & $3(15.0)$ & $3(17.6)$ & $1(7.1)$ & $1(4.5)$ & 0 & 0 \\
\hline Timepoint 2 & & $n=112$ & $n=20$ & $\mathrm{n}=17$ & $n=14$ & $n=22$ & $n=27$ & $\mathrm{n}=12$ \\
\hline \multirow[t]{4}{*}{ Primary Sedatives [no.(\%)] } & Promazine & $5(4.4)$ & $2(10.0)$ & 0 & $1(7.1)$ & $1(4.5)$ & $1(3.7)$ & 0 \\
\hline & Temazepam & $4(3.6)$ & 0 & 0 & $1(7.1)$ & $1(4.5)$ & 0 & $2(16.7)$ \\
\hline & Zopiclone & $4(3.6)$ & $1(5.0)$ & 0 & 0 & 0 & $2(7.4)$ & $1(8.3)$ \\
\hline & Lorazepam & $3(2.7)$ & 0 & 0 & $1(7.1)$ & $1(4.5)$ & $1(3.7)$ & 0 \\
\hline \multirow{4}{*}{$\begin{array}{l}\text { Drugs with sedation as a prominent adverse effect/ } \\
\text { containing a sedating component [no.(\%)] }\end{array}$} & Citalopram & $20(17.9)$ & $6(30.0)$ & $1(5.9)$ & $1(7.1)$ & $1(4.5)$ & $6(22.2)$ & $5(41.7)$ \\
\hline & Trazodone & 15(13.4) & 0 & 0 & $3(21.4)$ & $11(50.0)$ & $1(3.7)$ & 0 \\
\hline & Quetiapine & $11(9.8)$ & 0 & $5(29.4)$ & 0 & $5(22.7)$ & $1(3.7)$ & 0 \\
\hline & Fluoxetine & $9(8.0)$ & $3(15.0)$ & $3(17.6)$ & $1(7.1)$ & $1(4.5)$ & $1(3.7)$ & 0 \\
\hline Timepoint 3 & & $\mathrm{n}=105$ & $\mathrm{n}=19$ & $n=16$ & $n=14$ & $\mathrm{n}=21$ & $n=25$ & $\mathrm{n}=10$ \\
\hline \multirow[t]{4}{*}{ Primary Sedatives [no.(\%)] } & Promazine & $7(6.7)$ & $2(10.0)$ & 0 & $1(7.1)$ & $1(4.8)$ & $2(8.0)$ & 0 \\
\hline & Temazepam & $3(2.9)$ & 0 & 0 & $1(7.1)$ & $1(4.8)$ & 0 & $1(8.3)$ \\
\hline & Zopiclone & $3(2.9)$ & 0 & 0 & 0 & 0 & $2(8.0)$ & 0 \\
\hline & Lorazepam & $3(2.9)$ & $2(10.0)$ & 0 & $1(7.1)$ & $1(4.8)$ & $1(4.0)$ & 0 \\
\hline \multirow{4}{*}{$\begin{array}{l}\text { Drugs with sedation as a prominent adverse effect/ } \\
\text { containing a sedating component [no.(\%)] }\end{array}$} & Citalopram & $18(15.7)$ & $5(26.0)$ & 0 & $2(14.3)$ & $1(4.8)$ & $5(20.7)$ & $5(50.0)$ \\
\hline & Trazodone & $15(14.3)$ & 0 & 0 & $3(21.4)$ & $10(47.6)$ & $1(4.0)$ & $1(10.0)$ \\
\hline & Quetiapine & 9 (8.6) & 0 & $4(25.0)$ & 0 & $4(19.0)$ & $1(4.0)$ & 0 \\
\hline & Fluoxetine & $8(7.6)$ & $3(15.8)$ & $2(12.5)$ & $1(7.1)$ & $1(4.8)$ & $1(4.0)$ & 0 \\
\hline
\end{tabular}

BL - baseline, TP - time point.

not used by residents in care home 2 , while residents in care homes 1 and 6 did not use anxiolytics (Table 4).

At time-points 2 and 3, the study population declined to 112 and to 105 participants respectively due to drop-out and death. The demographic data, prevalence of psychotropic drug use, mean number of regular medications and prevalence of polypharmacy are presented in Tables 1 and 2 and are similar to those presented at baseline, although at time-point 3 , there was a significant difference in mean age of the residents in care homes 3 (90.4 years) and 5 (83.2 years) [Kruskal-Wallis test, $\mathrm{p}=0.02$ ]. At time-points 2 and 3, as at baseline, the most commonly used atypical antipsychotic was quetiapine (Table 5). At time-point 2, conventional antipsychotics were not used by residents in care home 2 and atypical antipsychotics were not used by residents in care homes 1, 3 and 6 . In care homes 4 and 5 both conventional and atypical antipsychotics were used, with atypical antipsychotics constituting the majority of antipsychotic use. SSRIs were the most commonly used antidepressants, with use ranging from $9.1 \%$ in care home 4 to $55.0 \%$ in care home 1 (Table 4 ). Table 5 shows that the most frequently administered hypnotics were temazepam and zopiclone (each at 3.6\%), while lorazepam was the most regularly used anxiolytic medication (2.7\%).

At time-point 3, variation in the use of antipsychotics was apparent within the population (Table $3, \chi 2, \mathrm{p}=0.04$ ). Moreover, antipsychotics were not administered on a regular basis for any resident in care home 6. Conventional antipsychotics were used in care homes $1,3,4$ and 5 but not in care homes 2 and 6. Promazine was the most frequently administered conventional antipsychotic (6.7\%). Use of antidepressants ranged from $25.0 \%$ in care home 2 to $64.3 \%$ in care home 3 (Table 3 ). As at baseline and time-point 2, SSRIs were the most frequently used antidepressants ranging from $9.5 \%$ in care home 4 to $52.6 \%$ in care home 1 (Table 4). Tricylic antidepressants were not used in care homes 1,4 or 6 . In care home 2, hypnotics and anxiolytics were not administered (Table 5). Furthermore, it is 
important to note that throughout the duration of this study, use of medications which are recognised as having prominent sedative adverse effects and/or containing sedative components outweighed the regular use of primary sedatives (Table 5).

\section{Discussion}

This study analysed the sedative load of medications regularly taken by older people with dementia living in six residential care homes in England, using the sedative load model $[35,36]$. This model has previously been used to examine the sedative load for residents with and without dementia in long-term care wards [33,34]. However, prior to the current study, it has not been used in settings where there is no on-site clinician.

The study population was more frequently administered medications with sedation as a prominent adverse effect or preparations with a sedating component rather than primary sedatives. Psychotropic medications were used on a regular basis by approximately two-thirds of residents in this study. This complements studies in nursing homes which have reported that $67 \%$ to $78 \%$ of patients with dementia were prescribed at least one psychotropic medication $[21,45,46]$.

Despite the variation in prescribing, sedative load scores were similar for residents across care homes at each time-point. These scores were lower than have been previously reported in residents of long-term care facilities and home-dwelling older people [34,35]. However, the findings of this study support a US study of long-term care facilities, in which residents with dementia were found to have a low sedative load [26].

Whilst some studies have reported that patients with dementia are frequently prescribed antipsychotics and hypnotics [20,47], this study found that across all care homes, antidepressants were more frequently used by residents $(49.8 \%)$, with antipsychotics $(19.0 \%)$ and hypnotics and anxiolytics (11.7\%) less routinely administered. However, this difference may in part by explained by the fact that in this study medications used on a pro re nata (prn) basis were excluded, whilst previous studies $[20,47]$ included these medications.

The most commonly used antidepressants in this study were SSRIs with citalopram being the most regularly used. This supports the findings from previous studies $[34,46]$. Fluoxetine was administered to $15 \%$ of the residents throughout the study period, despite its long halflife which may make it inappropriate for use in older people [48]. In care home 4, SSRIs were less commonly used and trazodone use predominated, which may be explained by an association between this care home and a memory clinic. Across the study period, regular use of hypnotics (ranging from $0 \%$ to $25.0 \%$ ) was similar to previous studies, with regular use of anxiolytics lower than previously reported $[21,34]$.

At $19.0 \%$, the prevalence of antipsychotic use among residents with dementia in this study was lower than previously reported in nursing home residents $[21,34,46]$. Overall, atypical antipsychotics were more commonly used than conventional antipsychotics, reflecting prescribing trends and the evidence that these medications are superior in controlling the behavioural and psychological symptoms of dementia [49], contribute less to the sedative load score and are associated with fewer extrapyramidal side-effects. However, care home 1 was a notable exception; throughout the study period, all residents taking antipsychotics used conventional antipsychotics. In care home 2 at baseline, antipsychotic use was significantly higher than in the other care homes. The resident characteristics may have contributed to this finding, since a high percentage of residents had three or more medical conditions recorded in their notes.

Previous studies have reported that the most frequently prescribed psychotropic medications for patients with dementia were antipsychotics $[34,46]$; however in this study antidepressants were predominantly used. This finding merits further investigation, using a larger population.

The underpinning knowledge for prescribing specific psychotropic medications and their impact on the symptoms and quality of life experienced by patients with dementia should be the focus of subsequent studies. Formal trials of psychotropic drugs with low sedative loading in care home residents with dementia should also be undertaken. Further research is also required into non-pharmacological alternatives to psychotropic medication, building upon the work conducted to date [50-63].

This study also highlights the lack of standardised diagnosis and staging of dementia in residential care home residents. One-quarter of the study participants did not have a diagnosis of dementia in their care home notes, but were determined by care home staff as having cognitive impairment consistent with a diagnosis of dementia. Furthermore, none of the care homes recorded severity of dementia in their care notes. There is a need for accurate and standardised diagnosis and staging of dementia in residential care homes to ensure that residents receive appropriate and timely care, despite the potential challenges such an approach may bring.

There are a number of methodological strengths of this study. The longitudinal design allowed examination of the pattern of medication use over the study period, while the use of an objective method to calculate the cumulative sedative effect of multiple medications eliminated subjective bias. Data collection directly from 
residents' medication administration records rather than from computerised data records facilitated the identification of medicines which were regularly administered rather than those which were dispensed but not actually taken by the patient.

It is also important to consider the methodological limitations of this study. Potential participants were identified either through a documented diagnosis of dementia within residents' care home notes or by senior care home staff as having cognitive impairment consistent with a diagnosis of dementia. The sedative load model presents inherent drawbacks including the exclusion of Group 3 drugs, which have the potential to cause sedation [36]. Furthermore, this model does not consider doses and frequencies of medications [34]. As with previous studies $[33,34]$, medications taken 'when required' (prn) were excluded from the analysis. These medications could further contribute to an individual's sedative load.

\section{Conclusions}

To optimise drug therapy for patients with dementia there is a need to take into consideration how individual drugs interact and contribute to the total sedative load score of the medications prescribed. In this study, residents with dementia were more frequently administered drugs which had sedation as a prominent adverse effect and/or contained a sedating component rather than primary sedatives.

Antidepressants, predominantly SSRIs, were most frequently administered, while levels of use of hypnotics and anxiolytics were lower. Sedative load scores were found to be similar throughout the study period for residents with dementia in each of the six care homes, and were lower than previously reported in other long-term care settings. This study focused on older people with dementia in care homes which have no on-site nursing or medical provision. It demonstrates the necessity for further research with this population and the need to develop interventions which support ongoing review and discussion between health service and care home providers. These interventions must recognise the complexities of working across systems of care with a workforce unqualified in medication review.

\footnotetext{
Acknowledgements

The authors would like to thank Dr Simon Bell for his advice regarding use of the sedative load model.

This article presents independent research commissioned by the National Institute for Health Research (NIHR) under its Programme Grants for Applied Research scheme (RP-PG-0606-1005). The views expressed in this publication are those of the author(s) and not necessarily those of the NHS, the NIHR or the Department of Health.
}

\section{Author details}

${ }^{1}$ School of Pharmacy, Queen's University Belfast, 97 Lisburn Road, Belfast, BT9 7BL, Northern Ireland, UK. ${ }^{2}$ Centre for Research in Primary and Community
Care, University of Hertfordshire, Hatfield, Hertfordshire, AL10 9AB, UK. ${ }^{3}$ General Practice \& Primary Care Research Unit, Institute of Public Health, Robinson Way, Cambridge, CB2 OSR, UK.

\section{Authors' contributions}

CP drafted the manuscript and participated in the analysis and interpretation of the data. JEH participated in the analysis and interpretation of the data. $N B, E M, I M$ and ES supported the data collection and analysis of the findings and commented on drafts of the manuscript. SA supported data analysis and final checks on data. CG designed the study and is the lead investigator, contributed sections of the manuscript and commented on all drafts. All authors read and approved the final manuscript.

\section{Competing interests}

The authors declare that they have no competing interests.

Received: 1 July 2011 Accepted: 30 September 2011

Published: 30 September 2011

\section{References}

1. Barber ND, Alldred DP, Raynor DK, Dickinson R, Garfield S, Jesson B, Lim R, Savage I, Standage C, Buckle P: Care homes' use of medicines study: prevalence, causes and potential harm of medication errors in care homes for older people. Qual Saf Health Care 2009, 18:341-346.

2. Goodman C, Davies SL: Good Practice outside the care homes. In Mental Health and Care Homes. Edited by: Dening T, Milne A. Oxford, Oxford University Press; 2011:297-312.

3. Hughes CM, Lapane $\mathrm{KL}$ : Administrative initiatives for reducing inappropriate prescribing of psychotropic drugs in nursing homes: how successful have they been? Drugs Aging 2005, 22:339-351.

4. Thomson MS, Gruneir A, Lee M, Baril J, Field TS, Gurwitz JH, Rochon PA: Nursing time devoted to medication administration in long-term care: clinical, safety, and resource implications. J Am Geriatr Soc 2009, 57:266-272.

5. Hajjar ER, Cafiero AC, Hanlon JT: Polypharmacy in elderly patients. Am J Geriatr Pharmacother 2007, 5:345-351.

6. Alldred DP, Zermansky AG, Petty DR, Raynor DK, Freemantle N, Eastaugh J, Bowie P: Clinical medication review by a pharmacist of elderly people living in care homes: pharmacist interventions. Int J Pharm Pract 2007, 15:93-99.

7. Hughes CM, Lapane K, Watson MC, Davies HTO: Does organisational culture influence prescribing in care homes for older people? A new direction for research. Drugs Aging 2007, 24:81-93.

8. Verrue $C L R$, Petrovic M, Mehuys $E$, Remon JP, Vander Stichele R: Pharmacists Interventions for Optimization of Medication Use in Nursing Homes: A Systematic Review. Drugs Aging 2009, 26:37-49.

9. Ganjavi H, Herrmann N, Rochon PA, Sharma P, Lee M, Cassel D, Freedman M, Black SE, Lanctôt KL: Adverse drug events in cognitively impaired elderly patients. Dement Geriatr Cogn Disord 2007, 23:395-400.

10. Oborne CA, Hooper R, Swift CG, Jackson SHD: Explicit, evidence-based criteria to assess the quality of prescribing to elderly nursing home residents. Age Ageing 2003, 32:102-108.

11. Fahey T, Montgomery AA, Barnes J, Protheroe J: Quality of care for elderly residents in nursing homes and elderly people living at home: controlled observational study. BMJ 2003, 326:580.

12. Ruths S, Straand J, Nygaard HA: Multidisciplinary medication review in nursing home residents: what are the most significant drug-related problems? The Bergen District Nursing Home (BEDNURS) study. Qual Saf Health Care 2003, 12:176-180.

13. Briesacher BA, Limcangco MR, Simoni-Wastila L, Doshi JA, Levens SR, Shea DG, Stuart B: The quality of antipsychotic drug prescribing in nursing homes. Arch Inter Med 2005, 165:1280-1285.

14. Rochon PA, Stukel TA, Bronskill SE, Gomes T, Sykora K, Wodchis WP, Hillmer M, Kopp A, Gurwitz JH, Anderson GM: Variation in nursing home antipsychotic prescribing rates. Arch Intern Med 2007, 167:676-683.

15. Hosia-Randell H, Muurinen SM, Pitkälä KH: Exposure to potentially inappropriate drugs and drug-drug interactions in elderly nursing home residents in Helsinki, Finland: A cross-sectional study. Drugs Aging 2008, 25, 683-692. 
16. Kamble P, Chen H, Sherer J, Aparasu RR: Antipsychotic drug use among elderly nursing home residents in the United States. Am J Geriatr Pharmacother 2008, 6:187-197.

17. Mann E, Kopke S, Haastert B, Pitkälä K, Meyer G: Psychotropic medication use among nursing home residents in Austria: a cross-sectional study. BMC Geriatr 2009, 9:18

18. Giron MS, Forsell Y, Bernsten C, Thorslund M, Winblad B, Fastbom J: Psychotropic drug use in elderly people with and without dementia. Int J Geriatr Psychiatry 2001, 9:900-906.

19. Hosia-Randell H, Pitkälä K: Use of psychotropic drugs in elderly nursing home residents with and without dementia in Helsinki, Finland. Drugs Aging 2005, 22:793-800.

20. Hartikainen S, Rahkonen T, Kautiainen H, Sulkava R: Use of psychotropics among home-dwelling nondemented and demented elderly. Int J Geriatr Psychiatry 2003, 18:1135-1141

21. Selbaek $G$, Kirkevold $\varnothing$, Engedal $K$ : The prevalence of psychiatric symptoms and behavioural disturbances and the use of psychotropic drugs in Norwegian nursing homes. Int J Geriatr Psychiatry 2007, 22:843-849.

22. Cao YJ, Mager DE, Simonsick EM, Hilmer SN, Ling SM, Windham BG, Crentsil V, Yasar S, Fried LP, Abernethy DR: Physical and cognitive performance and burden of anticholinergics, sedatives, and ACE inhibitors in older women. Clin Pharmacol Ther 2008, 83:422-429.

23. Gnjidic D, Cumming RG, Le Couteur DG, Handelsman DJ, Naganathan V, Abernethy DR, Hilmer SN: Drug Burden Index and physical function in older Australian men. Br J Clin Pharmacol 2009, 68:97-105.

24. Hanlon JT, Boudreau RM, Roumani YF, Newman AB, Ruby CM, Wright RM, Hilmer SN, Shorr Rl, Bauer DC, Simonsick EM, Studenski SA: Number and dosage of central nervous system medications on recurrent falls in community elders: the Health, Aging and Body Composition Study. $J$ Gerontol A Biol Sci Med Sci 2009, 64:492-498.

25. Hilmer SN, Mager DE, Simonsick EM, Cao Y, Ling SM, Windham BG, Harris TB, Hanlon JT, Rubin SM, Shorr RI, Bauer DC, Abernethy DR: A drug burden index to define the functional burden of medication in older people. Arch Intern Med 2007, 167:781-787.

26. Sloane P, Ivey J, Roth $M$, Roederer M, Williams CS: Accounting for the sedative and analgesic effects of medication changes during patient participation in clinical research studies: measurement development and application to a sample of institutionalized geriatric patients. Contemp Clin Trials 2008, 29:140-148.

27. Wilson NM, Hilmer SN, March LM, Cameron ID, Lord SR, Seibel MJ, Mason RS, Chen JS, Cumming RG, Sambrook PN: Associations between drug burden index and falls in older people in residential aged care. $J$ Am Geriatr Soc 2011, 59:875-880.

28. Wright RM, Roumani YF, Boudreau R, Newman AB, Ruby CM, Studenski SA, Shorr RI, Bauer DC, Simonsick EM, Hilmer SN, Hanlon JT, and for the Health Aging and Body Composition Study: Effect of central nervous system medication use on decline in cognition in community-dwelling older adults: findings from the Health, Aging and Body Composition Study. J Am Geriatr Soc 2009, 57:243-250.

29. Boudreau RM, Hanlon JT, Roumani YF, Studenski SA, Ruby CM, Wright RM, Hilmer SN, Shorr RI, Bauer DC, Simonsick EM, Newman AB: Central nervous system medication use and incident mobility limitation in community elders: the Health, Aging, and Body Composition Study. Pharmacoepidemiol Drug Saf 2009, 18:916-92.

30. Hilmer SN, Mager DE, Simonsick EM, Ling SM, Windham BG, Harris TB, Shorr Rl, Bauer DC, Abernethy DR, Health ABC Study: Drug burden index score and functional decline in older people. Am J Med 2009, 122:1142-1149.

31. Wilson NM, Hilmer SN, March LM, Cameron ID, Lord SR, Seibel MJ, Sambrook PN: Associations between drug burden index and physical function in older people in residential aged care facilities. Age Ageing 2010, 39:503-507.

32. Taipale HT, Hartikainen S, Bell JS: A comparison of four methods to quantify the cumulative effect of taking multiple drugs with sedative properties. Am J Geriatr Pharmacother 2010, 8:460-471.

33. Taipale HT, Bell JS, Soini H, Pitkälä KH: Sedative load and mortality among residents of long-term care facilities: a prospective cohort study. Drugs Aging 2009, 26:871-881.

34. Bell JS, Taipale HT, Soini H, Pitkälä KH: Sedative load among long-term care facility residents with and without dementia: a cross-sectional study. Clin Drug Investig 2010, 30:63-70.
35. Linjakumpu TA, Hartikainen SA, Klaukka TJ, Koponen HJ, Hakko HH, Viilo KM, Haapea M, Kivelä SL, Isoaho RE: Sedative drug use in the home dwelling elderly. Ann Pharmacother 2004, 38:2017-2022.

36. Linjakumpu T, Hartikainen S, Klaukka T, Koponen H, Kivelä SL, Isoaho R: A model to classify the sedative load of drugs. Int J Geriatr Psychiatry 2003, 18:542-544.

37. Nishtala PS, Hilmer SN, McLachlan AJ, Hannan PJ, Chen TF: Impact of residential medication management reviews on Drug Burden Index in aged-care homes. Drugs Aging 2009, 26:677-686.

38. Nishtala PS, MCLachlan AJ, Bell JS, Chen TF: Determinants of antipsychotic medication use among older people living in aged care homes in Australia. Int J Geriatr Psychiatry 2010, 25:449-457.

39. Goodman C, Baron NL, Machen I, Stevenson E, Evans C, Davies SL, lliffe S: Culture, consent, costs and care homes: enabling older people with dementia to participate in research. Aging Ment Health 2011, 15:475-481.

40. WHO Collaborating Centre for Drug Statistics Methodology:: The Anatomical Therapeutic Chemical Classification System. Norwegian Institute of Public Health [online]; 2008 [http://www.whocc.no/ atc_ddd_index/], (accessed 24 January 2011)..

41. Folstein MF, Folstein S, McHugh PR: "Mini-mental state": a practical method for grading the cognitive state of patients for the clinician. $J$ Psychiatr Res 1975, 12:189-198.

42. Alexopoulos GS, Abrams RC, Young RC, Shamoian CA: Cornell scale for depression in dementia. Biol Psychiatry 1988, 23:271-284.

43. Department of Health:: National Service Framework for Older People. London: Department of Health; 2001 [http://www.dh.gov.uk/ prod_consum_dh/groups/dh_digitalassets/@dh/@en/documents/ digitalasset/dh_4067247.pdf], (accessed 3 February 2011).

44. Cohen-Mansfield J: Instruction Manual for the Cohen-Mansfield Agitation Inventory (CMAI). The Research Institute of the Hebrew Home of Greater Washington, Rockville; 1991.

45. Kim H, Whall AL: Factors associated with psychotropic drug usage among nursing home residents with dementia. Nurs Research 2006, 55:252-258.

46. Pitkala KH, Laurila JV, Strandberg TE, Tilvis RS: Behavioural symptoms and the administration of psychotropic drugs to aged patients with dementia in nursing homes and in acute geriatric wards. Int Psychogeriatr 2004, 16:61-74.

47. Giron MS, Forsell Y, Bernsten C, Thorslund M, Winblad B, Fastbom J: Psychotropic drug use in elderly people with and without dementia. Int J Geriatr Psychiatry 2001, 16:900-906.

48. Fick DM, Cooper JW, Wade WE, Waller JL, Maclean JR, Beers MH: Updating the Beers criteria for potentially inappropriate medication use in older adults: results of a US consensus panel of experts. Arch Intern Med 2003, 163:2716-2724.

49. Sink KM, Holden KF, Yaffe K: Pharmacological treatment of neuropsychiatric symptoms of dementia: a review of the evidence. JAMA 2005, 293:596-608.

50. Cohen-Mansfield J, Werner P: Management of verbally disruptive behaviours in nursing home residents. J Gerontol A Biol Sci Med Sci 1997, 52:M369-M377.

51. Allen-Berge R, Stevens AB, Burgio LD: Effective behavioural interventions for decreasing dementia-related challenging behavior in nursing homes. Int J Geriatr Psychiatry 1999, 14:213-228.

52. Turner S: Behavioural symptoms of dementia in residential settings: $A$ selective review of non-pharmacological interventions. Aging Ment Health 2005, 9:93-104.

53. Livingston G, Johnston K, Katona C, Paton J, Lyketsos CG, Old Age Task Force of the World Federation of Biological Psychiatry: Systematic review of psychological approaches to the management of neuropsychiatric symptoms of dementia. Am J Geriatr Psychiatry 2005, 196:1996-2021.

54. Fossey J, Ballard C, Juszczak E, James I, Alder N, Jacoby R, Howard R: Effect of enhanced psychosocial care on antipsychotic use in nursing home residents with severe dementia: cluster randomised trial. BMJ 2006, 332:756-758.

55. Cohen-Mansfield J, Libin A, Marx MS: Nonpharmacological treatment of agitation: a controlled trial of systematic individualized intervention. J Gerontol A Biol Sci Med Sci 2007, 62:908-916.

56. Ballard C, Brown R, Fossey J, Douglas S, Bradley P, Hancock J, James IA, Juszczak E, Bentham P, Burns A, Lindesay J, Jacoby R, O'Brien J, Bullock R, Johnson T, Holmes C, Howard R: Brief Psychosocial Therapy for the 
Treatment of Agitation in Alzheimer Disease (The CALM-AD Trial). Am J Geriatr Pharmacother 2009, 17:726-733.

57. Deudon A, Maubourguet N, Gervais X, Leone E, Brocker P, Carcaillon L, Riff S, Lavallart B, Robert PH: Non-pharmacological management of behavioural symptoms in nursing homes. Int I Geriatr Psychiatry 2009, 24:1386-1395.

58. Kong EH, Evans LK, Guevara JP: Nonpharmacological intervention for agitation in dementia: a systematic review and meta-analysis. Aging Ment Health 2009, 13:512-520.

59. O'Connor DW, Ames D, Gardner B, King M: Psychosocial treatments of behavior symptoms in dementia: a systematic review of reports meeting quality standards. Inter Psychogeriatr 2009, 21:225-240.

60. O'Connor DW, Ames D, Gardner B, King M: Psychosocial treatments of psychological symptoms in dementia: a systematic review of reports meeting quality standards. Int Psychogeriatr 2009, 21:241-251.

61. Vernooij-Dassen M, Vasse E, Zuidema S, Cohen-Mansfield J, Moyle W: Psychosocial interventions in dementia care in long term care. Int Psychogeriatr 2010, 22:1121-8.

62. Cohen-Mansfield J, Marx MS, Dakheel-Ali M, Regier NG, Thein K, Freedman L: Can agitated behavior of nursing home residents with dementia be prevented with the use of standardized stimuli? J Am Geriatr Soc 2010, 58:1459-1464.

63. Vasse E, Vernooij-Dassen M, Cantegril I, Franco M, Dorenlot P, Woods B, Moniz-Cook E: Guidelines for psychosocial interventions in dementia care: a European survey and comparison. Int J Geriatr Psychiatry 2011, 2 Mar 2011.

\section{Pre-publication history}

The pre-publication history for this paper can be accessed here: http://www.biomedcentral.com/1471-2318/11/56/prepub

doi:10.1186/1471-2318-11-56

Cite this article as: Parsons et al: Sedative load of medications prescribed for older people with dementia in care homes. BMC Geriatrics 2011 11:56.

\section{Submit your next manuscript to BioMed Central and take full advantage of:}

- Convenient online submission

- Thorough peer review

- No space constraints or color figure charges

- Immediate publication on acceptance

- Inclusion in PubMed, CAS, Scopus and Google Scholar

- Research which is freely available for redistribution

Submit your manuscript at www.biomedcentral.com/submit 